\title{
Macrophage Migration Inhibitory Factor as a Predictor for Long-term Improvements After Mindfulness-Based Group Therapy or Treatment as Usual for Depression, Anxiety or Stress and Adjustment Disorders
}

\author{
Kristina Sundquist $^{1,2} \cdot$ Karolina Palmér $^{1} \cdot$ Ashfaque A. Memon ${ }^{1} \cdot$ Jan Sundquist ${ }^{1,3} \cdot$ Xiao Wang $^{1}$ (I)
}

Published online: 13 March 2020

(C) The Author(s) 2020

\begin{abstract}
Objectives Identification of biological markers that can guide treatment selection is considered to be a viable solution for personalized treatment for patients with psychiatric disorders. This study investigated whether macrophage migration inhibitory factor (MIF) levels at baseline were associated with mindfulness-based group therapy or cognitive behavioral therapy response in patients with mild to moderate symptoms of depression, anxiety, or stress- and adjustment disorders.

Methods A total of 168 patients (aged 21-65 years) with psychiatric disorders were included from a randomized controlled trial. Plasma MIF levels in all the patients were analyzed using Luminex assay.

Results Higher MIF levels at baseline were significantly associated with better long-term (1-year follow-up) improvement in psychiatric symptoms, as measured by changes in the Montgomery-Åsberg Depression Rating Scale (MADRS-S), compared with lower MIF levels, after adjustment for baseline MADRS-S score, age, sex, BMI, and pharmacotherapy $(\beta=5.89, p=0.001)$. Patients with higher levels of MIF $(8235-23,391 \mathrm{pg} / \mathrm{ml})$ had an almost 6 points' larger decrease in MADRS-S score after 1 year compared with those with lower MIF (727-8223 pg/ml) at baseline. Similar trends were seen after 8 weeks, albeit non-significant $(\beta=1.99, p=0.18)$.

Conclusions The findings indicate that higher plasma MIF levels at baseline may predict better long-term outcomes with psychotherapeutic interventions for mild to moderate symptoms of depression, anxiety, or stress and adjustment disorders. MIF levels may serve as a potential biomarker that can guide treatment selection for the personalized treatment for patients with psychiatric disorders.
\end{abstract}

Keywords Macrophage migration inhibitory factor $\cdot$ Depression $\cdot$ Anxiety $\cdot$ Mindfulness-based therapy $\cdot$ Cognitive behavioral therapy

Electronic supplementary material The online version of this article (https://doi.org/10.1007/s12671-020-01352-3) contains supplementary material, which is available to authorized users.

Xiao Wang

xiao.wang@med.lu.se

1 Center for Primary Health Care Research, Lund University/Region Skåne, Inga-Marie Nilssons gata 53, Wallenberg Laboratory, Plan 6, Box 50332, 20213 Malmö, Sweden

2 Department of Family Medicine and Community Health, Department of Population Health Science and Policy, Icahn School of Medicine at Mount Sinai, New York, USA

3 Center for Community-based Healthcare Research and Education (CoHRE), Department of Functional Pathology, School of Medicine, Shimane University, Matsue, Japan
The ultimate goal concerning the treatment of patients with psychiatric disorders should be to achieve full remission. However, in clinical studies, only about one-third of patients achieve remission after the initial stage of treatment (Toups et al. 2011; Tranter et al. 2002). Precision, or personalized medicine, is one of the strategic plans of the National Institute of Mental Health that aims to improve treatment outcomes regarding mental health (Insel et al. 2010). Improved prediction of treatment response could have many benefits for patients and reduce health care costs; this is a key goal of the precision medicine movement (Auffray and Hood 2012; McMahon 2014; Wallace et al. 2013). It is still a big challenge to select specific antidepressant treatments or non-pharmacological treatments, such as psychological interventions for a given patient. Therefore, identification of biological markers 
that can guide treatment selection is considered to be a viable solution for the personalized treatment of patients with psychiatric disorders.

Psychological interventions, such as mindfulness-based group therapy (MBGT) or cognitive behavioral therapy (CBT), are effective ways for improving the effects of psychological symptoms and reducing proinflammatory processes (Black and Slavich 2016; Irwin et al. 2015; Khoury et al. 2013; Memon et al. 2017; Sundquist et al. 2015). Sundquist et al. (2015) found that an 8-week mindfulness-based group therapy or treatment as usual (TAU) (mainly CBT) had clinically meaningful effects on patients with depression and anxiety, and this effect persisted even at the 1-year follow-up (Sundquist et al. 2019). The interventions have also demonstrated a concomitant reduction in inflammatory cytokine concentrations after 8 weeks of treatment and depressive symptoms after 8 weeks of treatment and 1-year follow-up. However, not all patients benefitted from the treatment (Sundquist et al. 2015; Sundquist et al. 2019). Therefore, it is important to identify biomarkers that can predict response for more tailored therapy. The baseline levels of C-reactive protein (CRP) and other circulating inflammatory markers, i.e., cytokines, have been proposed to serve as potential biomarkers for predicting the response of antidepressants in patients with depressive disorders (Cattaneo et al. 2016; Howren et al. 2009). However, information on biomarkers to predict response to psychotherapeutic interventions in patients with depression or anxiety is scarce. In a recent report, Memon et al. (2017) assessed both CRP and the cytokine IL-8 as potential predictive biomarkers for MBGT or TAU, but none was predictive; this suggests that other biomarkers should be explored.

Macrophage migration inhibitory factor (MIF) is one of the first cytokine-like proteins that was discovered more than 50 years ago (Bloom and Bennett 1966; David 1966). MIF was named for its ability to recruit macrophages, which have an important role in the immune system, to sites of inflammation and prevent random migration of macrophages. MIF can be released from almost all white blood cells and expressed in the brain tissue as well. Furthermore, MIF has been involved in many biological processes and acts as a central regulator of inflammatory responses (Bernhagen et al. 2007; Calandra and Roger 2003; Mitchell et al. 1999; Savaskan et al. 2012). Several disease conditions, such as cardiovascular disease (Zernecke et al. 2008) and diabetes (Toso et al. 2008), have also been associated with MIF. Circulating MIF is increased during episodes of inflammation, infection, and stress (Beishuizen et al. 2001; Calandra and Roger 2003).

Dysregulation of the immune system is associated with the pathophysiology of certain psychiatric disorders, such as depression (Irwin and Miller 2007). The role of MIF in the pathobiology of depression has been investigated in various ways (Bloom and Al-Abed 2014). Animal studies have shown that MIF is expressed in the brain especially in the areas concerning behavioral symptoms of depression and anxiety (Conboy et al. 2011). Genetic deletion of MIF has resulted in increased anxiety- and depression-like behaviors and the role of MIF in mediating the antidepressant function of exercise has been found (Conboy et al. 2011; Moon et al. 2012). Previous studies have shown that blood MIF levels are increased in subjects with mild to moderate depression, major depression, and other mood disorders (Baugh and Donnelly 2003; Bloom and Al-Abed 2014; Hawkley et al. 2007; Musil et al. 2011). Other studies have also shown that treatment with antidepressant or psychotherapeutic interventions in patients with psychiatric disorders can reduce the blood levels of MIF (Cattaneo et al. 2013; Wang et al. 2018b). Rethorst et al. (2013) reported that higher baseline levels of TNF- $\alpha$, another cytokine were associated with a more rapid drop in depressive symptoms over the course of the 12 -week exercise intervention.

The present study is one in an ongoing series reporting data from a single cohort of participants (Memon et al. 2017; Sundquist et al. 2015; Wang et al. 2015, 2018b). One of the general goals was to identify a biomarker that could help clinicians to select patients most likely to respond to psychotherapy. In the present study, the aim was to investigate the association between baseline MIF and MBGT/TAU response in patients with mild to moderate symptoms of depression, anxiety, or stress- and adjustment disorders. The hypothesis was that patients with different levels of MIF at baseline might respond differently to the therapeutic interventions.

\section{Methods}

\section{Participants}

The study population was based on data from a previously published randomized controlled trial (RCT) (ClinicalTrials. gov: NCT01476371). A total of 168 patients with depression, anxiety, or stress- and adjustment disorders comprised the study population. All of the patients were recruited from the 16 primary health care centers that had participated in a RCT of mindfulness therapy compared with TAU. A detailed description of the study design is provided in the previous article (Sundquist et al. 2015). To summarize, patients were recruited between 4 January 2012 and 22 March 2012 at the 16 primary health care centers in urban and rural settings in Skåne, which is located in the southernmost part of Sweden. The inclusion criteria were as follows: (1) age 20-64 years; (2) ability to speak and read Swedish; and (3) a score of $\geq 10$ on the Patient Health Questionnaire (PHQ)-9 or $\geq 7$ on the Hospital Anxiety and Depression Scale (HADS) or a total score on the Montgomery-Åsberg Depression Rating Scale (MADRS-S) between 13 and 34 (mild to moderate depression). In addition, all clinical diagnoses were made by doctors at the 16 primary 
health care centers, including one or more of the following ICD-10 psychiatric diagnoses: F32.0, mild depressive episode; F32.1, moderate depressive episode; F32.9, depressive episode, unspecified; F33.0, recurrent depressive disorder, current episode mild; F33.1, recurrent depressive disorder, current episode moderate; F41.0, panic disorder; F41.1, generalized anxiety disorder; F41.2, mixed anxiety and depressive disorder; F41.3, other mixed anxiety disorders; F41.8, other specified anxiety disorders; F41.9, anxiety disorder, unspecified; F43.2, adjustment disorders; F43.8, other reactions to severe stress; and F43.9, reaction to severe stress, unspecified. The rationale for using multiple scales to assess symptoms of depression and anxiety was that different scales are used in clinical practice worldwide (Sundquist et al. 2015). Eligible patients had a clinical diagnosis according to the International Classification of Diseases (ICD)-10 criteria. All clinical diagnoses were made by doctors at the 16 participating primary health care centers in Sweden. The exclusion criteria were as follows: severe personality disorder (these patients should not be treated in primary health care), risk of suicide, pregnancy, thyroid disease, current psychotherapy of any kind, and participation in any other psychiatric intervention study. The characteristics of the study population are summarized in Table 1 . The mean age in the whole group $(n=168)$ was 41.9 years $(\mathrm{SD}=11)$ and most of the participants were women (87\%). The mean body mass index (BMI) was 27.0. The median MADRS-S score at baseline was 20; this score indicates mild to moderate symptoms of depression and/or anxiety.

\section{Procedures}

Each participant was randomly allocated to either the MBGT or TAU group, and they were not allowed to change from their assigned group during the follow-up. The mindfulness treatment was provided by certified mindfulness instructors. In each primary health care centers, two mindfulness instructors were present at each group session. A maximum of ten participants were in each group. All the mindfulness instructors received the training program at our department (Center for Primary Health Care Research, Malmö, Sweden). The training was given for 6 days and all sessions were led by two experts in mindfulness education and therapy (Sundquist et al. 2015). A key part of the training is the instructors' own mindfulness training. The future instructors were trained in how to guide individuals and groups in mindfulness training so that the individual may develop a greater awareness of thoughts, feelings, and bodily sensations and thus be able to cope better with stress and difficulties in everyday life. All the instructors in the 6-day course completed the program, passed the oral examination, and became certified mindfulness instructors. The patients received antidepressants and anxiolytics (pharmacotherapy) if deemed necessary (Wang et al. 2018a).
Table 1 Characteristics of the study population at baseline $(n=168)$

\begin{tabular}{|c|c|}
\hline Variables & Patients $(n=168)$ \\
\hline \multicolumn{2}{|l|}{ Age, years } \\
\hline Mean (SD) & $41.9(11.0)$ \\
\hline \multicolumn{2}{|l|}{ Sex, $n(\%)$} \\
\hline Male & $22(13)$ \\
\hline Female & $146(87)$ \\
\hline \multicolumn{2}{|l|}{$\mathrm{BMI}^{\mathrm{a}}$} \\
\hline Mean $(\mathrm{SD})$ & $27.0(5.6)$ \\
\hline \multicolumn{2}{|l|}{ Antidepressants, $n(\%)^{\mathrm{b}}$} \\
\hline Yes & $50(33)$ \\
\hline No & $90(54)$ \\
\hline \multicolumn{2}{|l|}{ Anxiolytics, $n(\%)^{\mathrm{c}}$} \\
\hline Yes & $23(14)$ \\
\hline No & $110(65)$ \\
\hline \multicolumn{2}{|l|}{ Baseline MADRS-S } \\
\hline Median score (IQR) & $20(11)$ \\
\hline \multicolumn{2}{|c|}{ Follow-up (8-week) MADRS-S } \\
\hline Median score (IQR) & $11(10)$ \\
\hline \multicolumn{2}{|c|}{ Follow-up (1-year) MADRS-S } \\
\hline Median score (IQR) & $10(11)$ \\
\hline \multicolumn{2}{|l|}{ Baseline MIF (pg/ml) } \\
\hline Median score (IQR) & $5398(4339)$ \\
\hline \multicolumn{2}{|c|}{ Follow-up (8-week) MIF (pg/ml) } \\
\hline Median score (IQR) & $4561(3131)$ \\
\hline
\end{tabular}

Each patient filled in the three self-rated questionnaires mentioned above (i.e., PHQ-9, HADS-A/HADS-D, and MADRSS) at baseline, after 8 weeks of treatment, and at 1-year followup (121 patients completed the self-rated questionnaires at the 1 -year follow-up; i.e., the attrition rate was $28 \%$ ). Blood samples were collected at the same time as the assessment of selfrated symptoms at baseline and after 8 weeks of treatment.

\section{Measures}

Whole blood $(6 \mathrm{ml})$ was collected in EDTA tubes. Samples were centrifuged at $2000 \mathrm{~g}$ for $10 \mathrm{~min}$ at $4{ }^{\circ} \mathrm{C}$. Plasma MIF levels were determined using the bead-based multiplex assay for the Luminex platform (R\&D Systems Inc) based on the manufacturer's instructions. More details about the experiments were described in the previous article (Wang et al. 2018a). In brief, $80 \mu \mathrm{l}$ of plasma was diluted 1:2 in the dilution buffer and then incubated with an antibody-coated magnetic 
bead. Protein levels were measured using the Bio-Plex® ${ }^{\circledR}$ suspension array system and data were analyzed with Bio-Plex ManagerTM software (Version 4). Absolute concentrations in picogram per milliliter were calculated from a standard curve generated from eight serially diluted standards provided in the kit. The intra- and inter-assay coefficient of variation $(\mathrm{CV})$ rates were $3.8 \%$ and $4.4 \%$, respectively. Duplicate samples were assayed and all results were reported as means.

\section{Data Analyses}

The mean and SD for age and BMI and the median and interquartile range (IQR) for the scores and MIF level were assessed. Sex, antidepressant, and anxiolytics use are presented as percentages. Linear regression models were used to assess the association between MIF levels at baseline (continuously transformed with natural logarithm and categorized into quartiles) and change in MADRS-S score after 8 weeks and after 1 year (MADRS-S at baseline-MADRS-S at follow-up). The following potential confounders were considered: MADRS-S score at baseline, age, sex, BMI, and pharmacotherapy (antidepressants and/or anxiolytics). This association is also demonstrated in Fig. 1 where the mean decrease in MADRS-S score is shown over time in the highest quartile and the three lowest quartiles together. The same analysis was performed on the other three scales. Polynomial regression models were used to explore if the associations between MIF levels and change in scores were nonlinear. We achieved this by adding a quadratic term of MIF levels in the linear regression model. To be able to interpret the linear effect, we centered the exposure before squaring it. The centering also reduces the correlation between the linear and quadratic term.

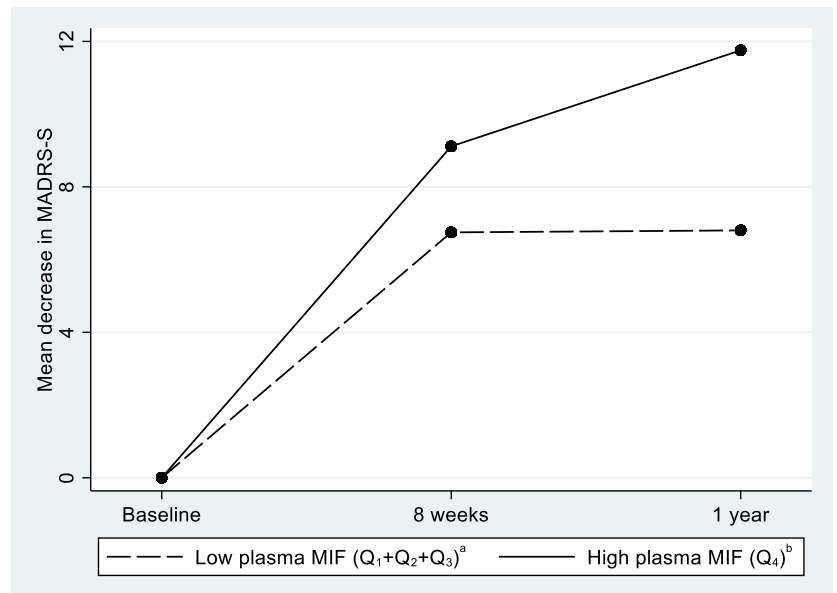

Fig. 1 Mean decrease in MADRS-S score after 8 weeks and after 1 year for low plasma MIF $\left(Q_{1}+Q_{2}+Q_{3}\right)$ and high plasma $\operatorname{MIF}\left(Q_{4}\right)$ at baseline. Linear regression models were used to assess the association between MIF levels at baseline and change in MADRS-S score after 8 weeks and after 1 year. $p=0.18$ ( 8 weeks) and $p=0.001$ ( 1 year). ${ }^{\mathrm{a}} Q_{1}=727$ $3861 \mathrm{pg} / \mathrm{ml}, Q_{2}=3920-5392 \mathrm{pg} / \mathrm{ml}, Q_{3}=5403-8223 \mathrm{pg} / \mathrm{ml} .{ }^{\mathrm{b}} Q_{4}=$ $8235-23,391 \mathrm{pg} / \mathrm{ml}$.
In addition, we explored the association by categorizing MIF levels into smaller groups to identify individuals most likely to respond to psychotherapy.

In a sensitivity analysis, we analyzed the interaction between MIF levels and treatment groups, i.e., whether the possible association between MIF levels at baseline and MADRS-S score differed between treatment groups, i.e., MBGT or TAU. We achieved this by adding an interaction term in the linear regression models.

Statistical analyses were performed by using IBM SPSS Statistics 23 (IBM, Armonk, NY, USA) and STATA version 15 (StataCorp LP).

\section{Results}

The median scores decreased significantly from 20 at baseline to 11 and 10 at 8 weeks of treatment and at the 1 -year followup, respectively (Table 1). In a previous article, we (Wang et al. 2018a) showed that MIF levels decreased significantly after 8 weeks of treatment (median 4561, IQR 3131, pg/ml) compared with baseline levels (median 5398, IQR 4339, pg/ $\mathrm{ml})(p<0.0001)$; there was no significant difference in patients treated with MBGT or TAU. Baseline levels of MIF were significantly associated with age $(\beta=0.006, p=0.001)$, but not with sex, BMI, or baseline MADRS-S score.

In the present study, we first performed a linear regression analysis to evaluate the potential association between MIF levels (as a continuous variable) at baseline and the improvement in psychiatric symptoms in response to the treatment (as measured by the change in MADRS-S scores) after 8 weeks and after 1 year (Table 2). Higher MIF levels at baseline were significantly associated with better improvement in psychiatric symptoms after 1-year follow-up, adjusted for baseline MADRS-S score $(\beta=2.56, p=0.02)$ and adjusted for baseline MADRS-S score together with age, sex, BMI, and pharmacotherapy $(\beta=3.19, p=0.009)$. In the 8 -week follow-up, higher MIF levels at baseline were significantly associated only with a better improvement in psychiatric symptoms when adjusted for baseline MADRS-S score $(\beta=2.61, p=0.04)$, but not when adjusted for baseline MADRS-S score together with age, sex, BMI, and pharmacotherapy $(\beta=1.99, p=0.18)$ (Table 2). Furthermore, we performed linear regression by dividing baseline MIF levels into quartiles where each quartile had 42 patients. We used quartiles because of the skewed distribution of MIF levels, i.e., more patients with low levels and fewer patients with high levels of plasma MIF. Patients in the highest quartile $\left(Q_{4}\right)$ of plasma MIF decreased almost 6 points more $\left(\beta=5.76, p=0.004, Q_{4}\right.$ vs. $\left.Q_{1}\right)$ in MADRS-S score after 1-year follow-up compared with patients in the lowest quartile $\left(Q_{1}\right)$ (Table 2). However, patients in the second $\left(Q_{2}\right)$ or third quartile $\left(Q_{3}\right)$ did not show any significantly better improvement in MADRS-S score in comparison with those in 
Table 2 The association between change in MADRS-S score (MADRS-S baseline - MADRS-S follow-up) after 8 weeks and after 1 year and plasma MIF $(\mathrm{pg} / \mathrm{ml})$ at baseline (continuously transformed with natural logarithm and categorized into quartiles)

\begin{tabular}{|c|c|c|c|c|c|c|c|c|c|}
\hline \multirow[t]{2}{*}{ Variable } & \multicolumn{3}{|c|}{ Unadjusted } & \multicolumn{3}{|c|}{ Adjusted $^{\mathrm{a}}$} & \multicolumn{3}{|c|}{ Adjusted $^{\mathrm{b}}$} \\
\hline & Beta & $95 \% \mathrm{CI}$ & $p$ value $^{\mathrm{c}}$ & Beta & $95 \% \mathrm{CI}$ & $p$ value $^{\mathrm{c}}$ & Beta & $95 \% \mathrm{CI}$ & $p$ value $^{\mathrm{c}}$ \\
\hline \multicolumn{10}{|c|}{ Outcome: change after 8 weeks } \\
\hline MIF cont ${ }^{\mathrm{d}}$ & 0.31 & $-1.95 ; 2.58$ & 0.78 & 0.84 & $-1.06 ; 2.73$ & 0.39 & 0.57 & $-1.58 ; 2.72$ & 0.60 \\
\hline$Q_{2}$ vs. $Q_{4}$ & -2.49 & $-6.08 ; 1.10$ & 0.17 & -1.93 & $-4.93 ; 1.08$ & 0.21 & -0.93 & $-4.32 ; 2.46$ & 0.59 \\
\hline$Q_{3}$ vs. $Q_{1}$ & -3.31 & $-6.96 ; 0.35$ & 0.08 & -1.95 & $-5.02 ; 1.12$ & 0.21 & -1.60 & $-5.16 ; 1.95$ & 0.37 \\
\hline$Q_{4}$ vs. $Q_{1}$ & 0.40 & $-3.21 ; 4.01$ & 0.83 & 1.29 & $-1.74 ; 4.31$ & 0.40 & 1.15 & $-2.41 ; 4.71$ & 0.52 \\
\hline$Q_{4}$ vs. $\left(Q_{1}+Q_{2}+Q_{3}\right)^{\mathrm{e}}$ & 2.36 & $-0.55 ; 5.28$ & 0.11 & 2.61 & $0.18 ; 5.04$ & 0.04 & 1.99 & $-0.90 ; 4.87$ & 0.18 \\
\hline \multicolumn{10}{|c|}{ Outcome: change after 1 year } \\
\hline MIF cont ${ }^{\mathrm{d}}$ & 2.48 & $0.03 ; 4.92$ & 0.05 & 2.56 & $0.37 ; 4.76$ & 0.02 & 3.19 & $0.80 ; 5.58$ & 0.009 \\
\hline$Q_{2}$ vs. $Q_{1}$ & -1.58 & $-5.59 ; 2.43$ & 0.44 & -1.03 & $-4.65 ; 2.59$ & 0.58 & 0.93 & $-2.87 ; 4.74$ & 0.63 \\
\hline$Q_{3}$ vs. $Q_{1}$ & -1.82 & $-6.00 ; 2.37$ & 0.39 & -1.34 & $-5.11 ; 2.43$ & 0.48 & -1.63 & $-5.66 ; 2.40$ & 0.42 \\
\hline$Q_{4}$ vs. $Q_{1}$ & 3.90 & $-0.01 ; 7.81$ & 0.05 & 3.90 & $0.37 ; 7.42$ & 0.03 & 5.76 & $1.86 ; 9.66$ & 0.004 \\
\hline$Q_{4}$ vs. $\left(Q_{1}+Q_{2}+Q_{3}\right)$ & 4.95 & $1.71 ; 8.20$ & 0.003 & 4.62 & $1.70 ; 7.54$ & 0.002 & 5.89 & $2.61 ; 9.18$ & 0.001 \\
\hline
\end{tabular}

the lowest quartile $\left(Q_{1}\right)$, which suggests that patients in the lower MIF quartiles $\left(Q_{1-3}\right)$ had a similar effect. Therefore, we combined patients in $Q_{1}, Q_{2}$, and $Q_{3}$ into one group and compared them with those in $Q_{4}$. Patients in the $Q_{4}$ group decreased almost 2 points more in MADRS-S score after 8 weeks of treatment $\left(\beta=1.99, p=0.18, Q_{4}\right.$ vs. $\left.Q_{1}+Q_{2}+Q_{3}\right)$ and almost 6 points more after 1 year compared with those in lower quartiles $\left(\beta=5.89, p=0.001, Q_{4}\right.$ vs. $\left.Q_{1}+Q_{2}+Q_{3}\right)$ (Table 2). This is also presented in a graph in Fig. 1 where the mean decrease in the two groups is shown over time. We also performed the same analysis on all four scales and the results were similar to the results from the MADRS-S score (data not shown). In addition, we performed a polynomial regression with both linear and quadratic effects in the models. Table S1 shows that the effect of plasma MIF levels at baseline seems to be nonlinear.

This was also confirmed in a likelihood ratio test that showed that adding a quadratic term in the linear regression models significantly improved the model fit both at 8 weeks and 1 year ( $p=0.01$ and $p=0.02$, respectively). In the 8-week model, the quadratic effect was significant with a positive sign before adjusting. This means that the curve is convex, with higher effect on the decrease in MADRS-S score for both lower levels $\left(Q_{1}\right)$ and higher levels of plasma MIF levels $\left(Q_{4}\right)$ at baseline. However, after adjustment, the effect became non-significant. In the adjusted 1-year model, both the linear and quadratic effects are significant. These results suggest that the potential effect on the improvement in the MADRS-S score differs by MIF levels with small or no effects for lower levels and high effects for higher levels. The graphs in Fig. $\mathrm{S} 1 \mathrm{a}$ and S1b display the curvilinear pattern of the association.

The highest quartile in all analyses showed better improvement. Baseline MIF levels were then categorized into subgroups (data not shown) to identify patients most likely to respond to psychotherapy. We were able to identify a group of 21 patients where all decreased their MADRS-S score after 1 year of follow-up (Fig. S2) and they had the highest levels of MIF at baseline (MIF $>11,120 \mathrm{pg} / \mathrm{ml}$ ).

We further examined the possible differences in the association between MIF at baseline and decrease in MADRS-S score between the groups (MBGT and TAU). We did this by adding an interaction between MIF levels at baseline and treatment group in the regression models (Table 2). This sensitivity analysis suggested that the mindfulness group had a slightly higher effect of high MIF levels than TAU, but there were no significant interactions (data not shown).

\section{Discussion}

In the present study, the findings were that higher MIF levels at baseline were significantly associated with better improvement in psychological symptoms at 1-year follow-up after the MBGT/TAU of depression, anxiety, and stress- and adjustment disorders. Similar trends were seen after 8 weeks but with a non-significant association. By stratification of the data 
according to the MIF levels at baseline, it was demonstrated that patients with the highest levels of MIF had the best improvement in their symptoms. The association was slightly higher in the mindfulness group compared with TAU, but not significant.

The results showed that patients with higher MIF at baseline benefitted more from psychotherapy than those with lower MIF. Therefore, MBGT/TAU may be more successful as a treatment in this subgroup, whereas other patients may not have the same response to MBGT/TAU. Concurring with the present results, one previous study has shown that inflammation biomarkers can modulate the effects of exercise on depression. Rethorst et al. (2013) found that higher baseline levels of the cytokine TNF- $\alpha$ were associated with a more rapid drop in depressive symptoms over the course of the 12 -week exercise intervention. In contrast to our finding, Cattaneo et al. (2016) reported that low MIF messenger RNA (mRNA) expression level at baseline was related to better outcomes with antidepressants in patients with depression. These contradictions in the results can be explained by the fact that protein levels do not necessarily represent the exact mRNA expression. There are many processes from mRNA to protein, which may affect the final levels of protein (Vogel and Marcotte 2012). For example, a combination of gene regulations (i.e., post-transcriptional, translational, and degradative) acting through miRNAs regulates protein levels (Mukherji et al. 2011). MiRNAs have been found to regulate protein levels, rather than to cause a large impact on mRNA levels (Baek et al. 2008).

The biological mechanisms underlying MIF's predictive effect are unclear. It seems that high levels of MIF may play a dual role in depression. On the one hand, MIF is a proinflammatory cytokine that is highly expressed in both immune cells and nonimmune cells including neurons in the brain tissues (Matsunaga et al. 1999). MIF is known to promote the expression of multiple cytokines and chemokines, such as IL6 and TNF- $\alpha$, which are believed to be involved in the pathophysiology of depression (Toh et al. 2006). On the other hand, in animal models, MIF also functions as an "antidepressant." Moreover, Conboy et al. (2011) also reported that MIF plays an important role in antidepressant-induced hippocampal neurogenesis. Subsequently, Moon et al. (2012) found that MIF itself could induce an antidepressive effect and mediate the antidepressant action of long-term exercise on depressive behavior in mice. In the present study, levels of MIF at baseline were associated with improvement in psychiatric symptoms after MBGT/TAU in a curvilinear pattern. These results suggest that MIF in this study may also have a dual role in response to psychotherapy. Other explanations behind the present findings could be that people with higher MIF levels at baseline are different from those with lower MIF levels, i.e., more active, have more outside psychosocial treatments and better social networks, and are healthier in other aspects.
Plasma MIF levels at baseline were significantly associated with improvement in psychiatric symptoms after 1-year of follow-up but the associations after 8 weeks of follow-up were non-significant. These results suggest that MIF levels at baseline may have stronger long-term than short-term effects in terms of response in depression/anxiety patients treated with psychotherapy. However, the mechanisms behind this association need to be disentangled.

\section{Limitations and Future Research}

Although the findings are promising, several limitations should be considered. First, we did not perform separate analyses according to the subtype of psychiatric disorders. However, overlapping symptoms are relatively common among these conditions and the patients were therefore analyzed together. Second, the present study did not include patients that did not receive psychotherapy as a control. It might be possible that the changes in protein were simply a reflection of variability over time. This study is more of a hypothesis generating study and the results need to be replicated in future studies. Third, we did not adjust for further potential treatment after the 8week intervention as we had no information about treatment after this time point in any of the two groups. Outside psychosocial treatment during the follow-up period may also have an effect on the improvement in psychiatric symptoms. We did, however, not collect data on outside psychosocial treatments during the follow-up period and are thus unable to consider this to be a potential confounder. Finally, the drop out of patients during 1-year follow-up may influence generalizability. The results indicated that higher plasma MIF levels at baseline may predict better long-term outcomes with psychotherapeutic interventions for mild to moderate symptoms of depression, anxiety, or stress- and adjustment disorders. These findings cannot, however, be used to guide clinical practice; they need to be confirmed in other settings.

Acknowledgments We would like to thank science editor Patrick Reilly for the critical reading of the manuscript as well as to biomedical analyst Hamideh Rastkhani for support in the lab.

Author Contributions KS: designed, wrote and edited the final manuscript. KP: analyzed the data, wrote and edited the final manuscript. AAM: collaborated with the design and executed the study, writing and editing the final manuscript. JS: collaborated with the design, writing and editing of the final manuscript. XW: designed and executed the study, contributed to the data analysis, wrote the paper

Funding Information Open access funding provided by Lund University. This project was supported by the Swedish Research Council as well as ALF funding from Region Skåne awarded to Jan Sundquist and Kristina Sundquist. 


\section{Compliance with Ethical Standards}

Competing Interests The authors declare that they have no conflict of interest.

Ethical Statement The study was performed according to the principles of the Declaration of Helsinki. It was reviewed and approved by the Ethics Committee of Lund University, prior to its commencement, on 5 October 2011 (application no. 2011/491). Written informed consent was obtained from all participants.

Open Access This article is licensed under a Creative Commons Attribution 4.0 International License, which permits use, sharing, adaptation, distribution and reproduction in any medium or format, as long as you give appropriate credit to the original author(s) and the source, provide a link to the Creative Commons licence, and indicate if changes were made. The images or other third party material in this article are included in the article's Creative Commons licence, unless indicated otherwise in a credit line to the material. If material is not included in the article's Creative Commons licence and your intended use is not permitted by statutory regulation or exceeds the permitted use, you will need to obtain permission directly from the copyright holder. To view a copy of this licence, visit http://creativecommons.org/licenses/by/4.0/.

\section{References}

Auffray, C., \& Hood, L. (2012). Editorial: systems biology and personalized medicine - the future is now. Biotechnology Journal, 7(8), 938-939. https://doi.org/10.1002/biot.201200242.

Baek, D., Villen, J., Shin, C., Camargo, F. D., Gygi, S. P., \& Bartel, D. P. (2008). The impact of microRNAs on protein output. Nature, 455(7209), 64-71. https://doi.org/10.1038/nature07242.

Baugh, J. A., \& Donnelly, S. C. (2003). Macrophage migration inhibitory factor: a neuroendocrine modulator of chronic inflammation. Journal of Endocrinology, 179(1), 15-23.

Beishuizen, A., Thijs, L. G., Haanen, C., \& Vermes, I. (2001). Macrophage migration inhibitory factor and hypothalamopituitary-adrenal function during critical illness. Journal of Clinical Endocrinology and Metabolism, 86(6), 2811-2816. https://doi.org/10.1210/jcem.86.6.7570.

Bernhagen, J., Krohn, R., Lue, H., Gregory, J. L., Zernecke, A., Koenen, R. R., Dewor, M., Georgiev, I., Schober, A., Leng, L., Kooistra, T., Fingerle-Rowson, G., Ghezzi, P., Kleemann, R., McColl, S. R., Bucala, R., Hickey, M. J., \& Weber, C. (2007). MIF is a noncognate ligand of CXC chemokine receptors in inflammatory and atherogenic cell recruitment. Nature Medicine, 13(5), 587-596. https://doi. org/10.1038/nm1567.

Black, D. S., \& Slavich, G. M. (2016). Mindfulness meditation and the immune system: a systematic review of randomized controlled trials. Annals of the New York Academy of Sciences, 1373(1), 13-24. https://doi.org/10.1111/nyas.12998.

Bloom, J., \& Al-Abed, Y. (2014). MIF: mood improving/inhibiting factor? Journal of Neuroinflammation, 11, 11. https://doi.org/10.1186/ 1742-2094-11-11.

Bloom, B. R., \& Bennett, B. (1966). Mechanism of a reaction in vitro associated with delayed-type hypersensitivity. Science, 153(3731), 80-82. https://doi.org/10.1126/science.153.3731.80.

Calandra, T., \& Roger, T. (2003). Macrophage migration inhibitory factor: a regulator of innate immunity. Nature Reviews Immunology, 3(10), 791-800. https://doi.org/10.1038/nri1200.

Cattaneo, A., Gennarelli, M., Uher, R., Breen, G., Farmer, A., Aitchison, K. J., Craig, I. W., Anacker, C., Zunsztain, P. A., McGuffin, P., \&
Pariante, C. M. (2013). Candidate genes expression profile associated with antidepressants response in the GENDEP study: differentiating between baseline 'predictors' and longitudinal 'targets'. Neuropsychopharmacology, 38(3), 377-385. https://doi.org/10. 1038/npp.2012.191.

Cattaneo, A., Ferrari, C., Uher, R., Bocchio-Chiavetto, L., Riva, M. A., \& Pariante, C. M. (2016). Absolute measurements of macrophage migration inhibitory factor and Interleukin-1-beta mRNA levels accurately predict treatment response in depressed patients. International Journal of Neuropsychopharmacology, 19(10). https://doi.org/10. 1093/ijnp/pyw045.

Conboy, L., Varea, E., Castro, J. E., Sakouhi-Ouertatani, H., Calandra, T., Lashuel, H. A., \& Sandi, C. (2011). Macrophage migration inhibitory factor is critically involved in basal and fluoxetine-stimulated adult hippocampal cell proliferation and in anxiety, depression, and memory-related behaviors. Molecular Psychiatry, 16(5), 533-547. https://doi.org/10.1038/mp.2010.15.

David, J. R. (1966). Delayed hypersensitivity in vitro: its mediation by cell-free substances formed by lymphoid cell-antigen interaction. Proceedings of the National Academy of Sciences of the United States of America, 56(1), 72-77. https://doi.org/10.1073/pnas.56.1. 72.

Hawkley, L. C., Bosch, J., Engeland, C. G., Marucha, P. T., \& Cacioppo, J. T. (2007). Loneliness, dysphoria, stress, and immunity: a role for cytokines. In N. P. Plotnikoff, R. E. Faith, A. J. Murgo, \& R. A. Good (Eds.), Cytokines: stress and immunity 2nd ed. (pp. 67-85). Boca Raton: CRC Press.

Howren, M. B., Lamkin, D. M., \& Suls, J. (2009). Associations of depression with C-reactive protein, IL-1, and IL-6: a meta-analysis. Psychosomatic Medicine, 71(2), 171-186. https://doi.org/10.1097/ PSY.0b013e3181907c1b.

Insel, T., Cuthbert, B., Garvey, M., Heinssen, R., Pine, D. S., Quinn, K., Sanislow, C., \& Wang, P. (2010). Research domain criteria (RDoC): toward a new classification framework for research on mental disorders. American Journal of Psychiatry, 167(7), 748-751. https:// doi.org/10.1176/appi.ajp.2010.09091379.

Irwin, M. R., \& Miller, A. H. (2007). Depressive disorders and immunity: 20 years of progress and discovery. Brain, Behavior, and Immunity, 21(4), 374-383. https://doi.org/10.1016/j.bbi.2007.01.010.

Irwin, M. R., Olmstead, R., Breen, E. C., Witarama, T., Carrillo, C., Sadeghi, N., Arevalo, J. M., Ma, J., Nicassio, P., Bootzin, R., \& Cole, S. (2015). Cognitive behavioral therapy and tai chi reverse cellular and genomic markers of inflammation in late-life insomnia: a randomized controlled trial. Biological Psychiatry, 78(10), 721729. https://doi.org/10.1016/j.biopsych.2015.01.010.

Khoury, B., Lecomte, T., Fortin, G., Masse, M., Therien, P., Bouchard, V., Chapleau, M. A., Paquin, K., \& Hofmann, S. G. (2013). Mindfulness-based therapy: a comprehensive meta-analysis. Clinical Psychology Review, 33(6), 763-771. https://doi.org/10. 1016/j.cpr.2013.05.005.

Matsunaga, J., Sinha, D., Pannell, L., Santis, C., Solano, F., Wistow, G. J., \& Hearing, V. J. (1999). Enzyme activity of macrophage migration inhibitory factor toward oxidized catecholamines. Journal of Biological Chemistry, 274(6), 3268-3271. https://doi.org/10.1074/ jbc.274.6.3268.

McMahon, F. J. (2014). Prediction of treatment outcomes in psychiatrywhere do we stand ? Dialogues in Clinical Neuroscience, 16(4), 455-464.

Memon, A. A., Sundquist, K., Ahmad, A., Wang, X., Hedelius, A., \& Sundquist, J. (2017). Role of IL-8, CRP and epidermal growth factor in depression and anxiety patients treated with mindfulness-based therapy or cognitive behavioral therapy in primary health care. Psychiatry Research, 254, 311-316. https://doi.org/10.1016/j. psychres.2017.05.012.

Mitchell, R. A., Metz, C. N., Peng, T., \& Bucala, R. (1999). Sustained mitogen-activated protein kinase (MAPK) and cytoplasmic 
phospholipase A2 activation by macrophage migration inhibitory factor (MIF). Regulatory role in cell proliferation and glucocorticoid action. Journal of Biological Chemistry, 274(25), 18100-18106. https://doi.org/10.1074/jbc.274.25.18100.

Moon, H. Y., Kim, S. H., Yang, Y. R., Song, P., Yu, H. S., Park, H. G., Hwang, O., Lee-Kwon, W., Seo, J. K., Hwang, D., Choi, J. H., Bucala, R., Ryu, S. H., Kim, Y. S., \& Suh, P. G. (2012). Macrophage migration inhibitory factor mediates the antidepressant actions of voluntary exercise. Proceedings of the National Academy of Sciences of the United States of America, 109(32), 13094-13099. https://doi.org/10.1073/pnas.1205535109.

Mukherji, S., Ebert, M. S., Zheng, G. X., Tsang, J. S., Sharp, P. A., \& van Oudenaarden, A. (2011). MicroRNAs can generate thresholds in target gene expression. Nature Genetics, 43(9), 854-859. https:// doi.org/10.1038/ng.905.

Musil, R., Schwarz, M. J., Riedel, M., Dehning, S., Cerovecki, A., Spellmann, I., Arolt, V., \& Muller, N. (2011). Elevated macrophage migration inhibitory factor and decreased transforming growth factor-beta levels in major depression-no influence of celecoxib treatment. Journal of Affective Disorders, 134(1-3), 217-225. https://doi.org/10.1016/j.jad.2011.05.047.

Rethorst, C. D., Toups, M. S., Greer, T. L., Nakonezny, P. A., Carmody, T. J., Grannemann, B. D., Huebinger, R. M., Barber, R. C., \& Trivedi, M. H. (2013). Pro-inflammatory cytokines as predictors of antidepressant effects of exercise in major depressive disorder. Molecular Psychiatry, 18(10), 1119-1124. https://doi.org/10.1038/mp.2012. 125.

Savaskan, N. E., Fingerle-Rowson, G., Buchfelder, M., \& Eyupoglu, I. Y. (2012). Brain miffed by macrophage migration inhibitory factor. International Journal of Cell Biology, 2012, 139573. https://doi. org/10.1155/2012/139573.

Sundquist, J., Lilja, A., Palmer, K., Memon, A. A., Wang, X., Johansson, L. M., \& Sundquist, K. (2015). Mindfulness group therapy in primary care patients with depression, anxiety and stress and adjustment disorders: randomised controlled trial. British Journal of Psychiatry, 206(2), 128-135. https://doi.org/10.1192/bjp.bp.114. 150243.

Sundquist, J., Palmer, K., Memon, A. A., Wang, X., Johansson, L. M., \& Sundquist, K. (2019). Long-term improvements after mindfulnessbased group therapy of depression, anxiety and stress and adjustment disorders: a randomized controlled trial. Early Intervention in Psychiatry, 13(4), 943-952. https://doi.org/10.1111/eip.12715.

Toh, M. L., Aeberli, D., Lacey, D., Yang, Y., Santos, L. L., Clarkson, M., Sharma, L., Clyne, C., \& Morand, E. F. (2006). Regulation of IL-1 and TNF receptor expression and function by endogenous macrophage migration inhibitory factor. Journal of Immunology, 177(7), 4818-4825. https://doi.org/10.4049/jimmunol.177.7.4818.

Toso, C., Emamaullee, J. A., Merani, S., \& Shapiro, A. M. (2008). The role of macrophage migration inhibitory factor on glucose metabolism and diabetes. Diabetologia, 51(11), 1937-1946. https://doi.org/ 10.1007/s00125-008-1063-3.

Toups, M. S., Greer, T. L., Kurian, B. T., Grannemann, B. D., Carmody, T. J., Huebinger, R., Rethorst, C., \& Trivedi, M. H. (2011). Effects of serum Brain Derived Neurotrophic Factor on exercise augmentation treatment of depression. Journal of Psychiatric Research, 45(10), 1301-1306. https://doi.org/10.1016/j.jpsychires.2011.05.002.

Tranter, R., O’Donovan, C., Chandarana, P., \& Kennedy, S. (2002). Prevalence and outcome of partial remission in depression. Journal of Psychiatry \& Neuroscience, 27(4), 241-247.

Vogel, C., \& Marcotte, E. M. (2012). Insights into the regulation of protein abundance from proteomic and transcriptomic analyses. Nature Reviews Genetics, 13(4), 227-232. https://doi.org/10.1038/ $\operatorname{nrg} 3185$.

Wallace, M. L., Frank, E., \& Kraemer, H. C. (2013). A novel approach for developing and interpreting treatment moderator profiles in randomized clinical trials. JAMA Psychiatry, 70(11), 1241-1247. https:// doi.org/10.1001/jamapsychiatry.2013.1960.

Wang, X., Sundquist, K., Hedelius, A., Palmer, K., Memon, A. A., \& Sundquist, J. (2015). Circulating microRNA-144-5p is associated with depressive disorders. Clinical Epigenetics, 7, 69. https://doi. org/10.1186/s13148-015-0099-8.

Wang, X., Sundquist, K., Palmer, K., Hedelius, A., Memon, A. A., \& Sundquist, J. (2018a). Macrophage migration inhibitory factor and microRNA-451a in response to mindfulness-based therapy or treatment as usual in patients with depression, Anxiety or Stress- and Adjustment Disorders. The International Journal of Neuropsychopharmacology. https://doi.org/10.1093/ijnp/pyy001.

Wang, X., Sundquist, K., Palmer, K., Hedelius, A., Memon, A. A., \& Sundquist, J. (2018b). Macrophage migration inhibitory factor and microRNA-451a in response to mindfulness-based therapy or treatment as usual in patients with depression, anxiety, or stress and adjustment disorders. International Journal of Neuropsychopharmacology, 21(6), 513-521. https://doi.org/10. 1093/ijnp/pyy001.

Zernecke, A., Bernhagen, J., \& Weber, C. (2008). Macrophage migration inhibitory factor in cardiovascular disease. Circulation, 117(12), 1594-1602. https://doi.org/10.1161/circulationaha.107.729125.

Publisher's Note Springer Nature remains neutral with regard to jurisdictional claims in published maps and institutional affiliations. 